\title{
AN HST SURVEY OF CORES OF EARLY-TYPE GALAXIES ${ }^{1}$
}

\section{JOHN KORMENDY ${ }^{2}$ AND YONG-IK BYUN}

Institute for Astronomy, University of Hawaii, 2680 Woodlawn Dr., Honolulu, HI 96822

E. A. AJHAR AND TOD R. LAUER

Kitt Peak National Observatory, National Optical Astronomy Observatories, P.O. Box 26732, Tucson, AZ 85726

ALAN DRESSLER

Carnegie Observatories, 813 Santa Barbara St., Pasadena, CA 91101

S. M. FABER AND CARL GRILLMAIR

UCO/Lick Observatory, University of California, Santa Cruz, CA 95064

KARL GEBHARDT AND DOUGLAS RICHSTONE

Dept. of Astronomy, Univ. of Michigan, Ann Arbor, MI 48109

AND

SCOTT TREMAINE

Canadian Institute for Theoretical Astrophysics, University of Toronto, 60 St. George St., Toronto M5S 1A7, Canada

\begin{abstract}
Photometry of the central parts of bulges and elliptical galaxies with the Hubble Space Telescope (HST) confirms and extends ground-based results. Most giant ellipticals have cuspy cores: at the "break radius" $r_{b}$ (formerly the core radius $r_{c}$ ), the steep outer surface brightness profile turns down to a shallow inner power law $I(r) \propto r^{-\gamma}, 0 \leq \gamma \lesssim 0.25$. The corresponding slope of the deprojected profile is derived; the flattest cores allow box orbits to survive. Cores continue to satisfy fundamental plane

${ }^{1}$ Based on observations with the NASA/ESA Hubble Space Telescope, obtained at the Space Telescope Science Institute, which is operated by AURA, Inc., under NASA contract NAS 5-26555.

${ }^{2}$ Visiting Astronomer at the Canada-France-Hawaii Telescope (CFHT), operated by the National Research Council of Canada, the Centre National de la Recherche Scientifique of France, and the University of Hawaii.
\end{abstract}


parameter correlations like those found from the ground. In particular, HST confirms that the luminosity sequence of elliptical galaxies (from cDs to M 32) is physically unrelated to spheroidal galaxies like Fornax. The latter are closely related to late-type dwarfs. Low-luminosity ellipticals do not show cores: $0.5 \lesssim \gamma \lesssim 1.3$. The most important new result is that global and core properties both show signs of a dichotomy between (i) low-luminosity ellipticals that rotate rapidly, that are nearly isotropic and oblate-spheroidal, that have disky-distorted isophotes, and that are coreless and (ii) giant ellipticals that are essentially nonrotating, anisotropic, and moderately triaxial, that are boxy-distorted, and that have cuspy cores.

Key words: Galaxies: Nuclei - Galaxies: Photometry - Galaxies: Structure

\section{Introduction}

The study of galaxy cores is a prime mission of HST. High-resolution photometry has now been published by a number of groups (Lauer et al. 1991; 1992a, b; 1993; 1995; Crane et al. 1993; Stiavelli et al. 1993; Kormendy et al. 1994; Grillmair et al. 1994; Forbes 1994; Forbes et al. 1994, 1995; Jaffe at al. 1994; van den Bosch et al. 1994; Ferrarese et al. 1994). This paper focuses on the work of our group; results from the other groups are similar. HST has enriched our understanding of galaxy cores; it has settled some outstanding issues, and it has provided a few surprises. But many results were already in place from ground-based photometry, and most of these have survived. We therefore begin with a brief review of ground-based work. We concentrate on one result that is particularly relevant at this meeting, i. e. the clear physical distinction between elliptical and spheroidal galaxies.

\section{Ground-Based Results: Elliptical and Spheroidal Galaxies as Distinct Families of Stellar Systems}

Ground-based work on galaxy cores is reviewed in Kormendy $(1982,1987 \mathrm{a})$ and in Kormendy \& Djorgovski (1989). The main results are:

1 - Cores: Most giant ellipticals have cores; i. e., central regions where the surface brightness profile $I(r)$ turns down from a steep $I \propto r^{-\beta}(\beta \sim 2)$ outer power law toward $I \simeq$ constant. The turndown is more gradual than in an isothermal sphere; this was demonstrated by the first CCD photometry (Young et al. 1978; Lauer 1985a, b) and is most convincingly seen in high-resolution photometry from the CFHT (Kormendy 1985a, 1987a) and NOT (Møller et al. 1995). The brightness profile is still rising where seeing becomes dominant, but ground-based photometry did not tell us the functional form of $I(r)$ at $r \ll r_{c}$. HST solves this problem $(\S 3)$. 
2 - Fundamental plane (FP) correlations: Lower-luminosity giant Es have smaller core radii $r_{c}$, higher central surface brightnesses $I_{0}$, and larger central velocity dispersions $\sigma$ (Kormendy 1984; 1985b; 1987a,b; Lauer 1985a, b; see Kormendy \& McClure 1993 and Kormendy \& Bender 1994 for recent versions). Bulges of disk galaxies are consistent with these correlations; when we speak of "ellipticals" below, we include bulges.

3 - Low-luminosity galaxies do not show resolved cores. Limits on $r_{c}$ are consistent with the FP relations, but there may be a dichotomy between coreless ellipticals with disky isophote distortions and boxy ellipticals with resolved cores (Nieto et al. 1991). This dichotomy is the subject of $\S 6$.

4 - Families of ellipsoidal stellar systems: The FP correlations of elliptical galaxies are very different from those of spheroidal (Sph) galaxies. For example, spheroidal galaxies with lower luminosities $L$ have lower core and effective surface brightnesses, while lower-luminosity ellipticals have higher surface brightnesses. The low-luminosity end of the $\mathrm{E}$ sequence is defined by M 32 and by similar $M_{V} \simeq-16$ ellipticals in Virgo, not by dwarf spheroidals like NGC 205, Fornax, or Draco. This was correctly postulated by Wirth \& Gallagher (1984) from remarkably meager statistics and then demonstrated by Kormendy $(1985 \mathrm{~b}, 1987 \mathrm{~b})$ using CFHT photometry of galaxies with a wide range in luminosities. The difference between $\mathrm{E}$ and Sph galaxies is global, not just a core property (Ichikawa et al. 1986, 1988; Kormendy 1987b; Binggeli \& Cameron 1991) ${ }^{3}$. Also, Wirth \& Gallagher (1984) suggested and Sandage et al. (1985), Binggeli (1987), Binggeli et al. (1988), and Ferguson \& Sandage (1991) showed that E and Sph galaxies have different luminosity functions. Ellipticals are bounded in luminosity. Objects like M 32 are rare; we are extremely fortunate to live so near a prototypical example. Spheroidals, on the other hand, begin to appear at $M_{B} \simeq-18$ and then have exponentially rising luminosity functions at faint magnitudes $M_{B}$.

Kormendy (1985b, 1987b) further showed that dwarf spheroidals are similar in global structure to dwarf spirals and irregulars. This almost certainly means that they are physically related. Binggeli (1994b) and Ferguson \& Binggeli (1994) review the possibilities. The relationship is complex; more than one physical process is likely to be be important even at a single luminosity. However, it is worth noting that about half of the Galaxy's dSph companions have stellar subpopulations that are $3-7$ Gy old (e.g., Da Costa 1992), so many dSph galaxies were Magellanic irregulars until relatively recently (Kormendy \& Bender 1994).

${ }^{3}$ Caution: Binggeli and collaborators call the galaxies in the Sph family "dwarf ellipticals" or "dEs" even though they are not related to ellipticals; see Binggeli (1994a) and Kormendy \& Bender (1994) for contrasting views on the terminology. We follow the Kormendy \& Bender convention. 
The "bottom line" is this: Sph galaxies are not ellipticals and probably formed differently from ellipticals. Compared to the difference between $\mathrm{E}$ and Sph galaxies, ellipticals are remarkably homogeneous in properties (Djorgovski \& Santiago 1993; Bender et al. 1993, 1994; Saglia et al. 1993; Djorgovski, Pahre, \& de Carvalho 1995), despite heterogeneous merger histories and even including the physical dichotomy discussed in $\S 6$.

\section{An HST Perspective on Galaxy Cores}

HST work on galaxy cores began with the S0 galaxy NGC 7457 (Lauer et al. 1991) and with the ellipticals M 87 and M 32 (Lauer et al. 1992a,b). NGC 7457 and M 32 have coreless power-law profiles, although limits on $r_{c}\left(0.05\right.$ and $0 !^{\prime \prime} 11$, respectively) are consistent with the FP correlations (Kormendy \& McClure 1993). In contrast, the core of M 87 was already well resolved from the ground (Kormendy 1985a). With HST, it is so well resolved that the nature of the inner profile becomes clear: inside a break radius $r_{b} \simeq r_{c}$, the steep outer power law turns down to a shallow inner power law, $I \propto r^{-0.26}$. These two types of profiles - power laws and cuspy cores - characterize almost all ellipticals (references in $\S 1$; Tremaine 1995).

Our own group obtained photometry for 45 galaxies in Cycles 1 and 2; Kormendy et al. (1994) present a preliminary report, and Lauer et al. (1995) publish the data in full. The images were Lucy - Richardson deconvolved; steep brightness profiles are accurate to $\sim 0.1 \mathrm{mag} \operatorname{arcsec}^{-2}$; core profiles are accurate to $\lesssim 0.05 \mathrm{mag} \operatorname{arcsec}^{-2}(\S 4)$.

Almost all high-luminosity galaxies have resolved cuspy cores like that of M 87. At small $r$, the profiles are shallow power laws, $I(r) \propto r^{-\gamma}$, with $0 \leq \gamma \lesssim 0.25$. A convenient parametrization is

$$
I(r)=I_{b} 2^{\frac{\beta-\gamma}{\alpha}}\left(\frac{r}{r_{b}}\right)^{-\gamma}\left[1+\left(\frac{r}{r_{b}}\right)^{\alpha}\right]^{\frac{\gamma-\beta}{\alpha}} .
$$

Here $r_{b}$ and $I_{b}$ replace the former parameters core radius $r_{c}$ and central surface brightness $I_{0}: r_{b}$ is the radius at which the steep outer $I \propto r^{-\beta}$ profile breaks into the shallow inner profile, and $I_{b}$ is the surface brightness at $r_{b}$. The parameter $\alpha$ measures the sharpness of the break. Fits of Equation 1 to the profiles are calculated in Byun et al. (1995); resulting core parameters are discussed in Faber et al. (1995) and in $\S 5$, below.

All of the low-luminosity galaxies except NGC $4486 \mathrm{~B}$ are unresolved. Like M 32, they have power-law profiles that remain steep $(0.5 \lesssim \gamma \lesssim 1.3)$ to radii $r<0$ " 1 . The division between galaxies with and without resolved cores occurs at $M_{V} \simeq-21 \pm 0.5$ but is not completely sharp ( $\left.\S 6\right)$.

A few galaxies show point sources added to core or power-law profiles. Some are active nuclei (NGC 6166: Filippenko, private communication). When we know or suspect that they are star clusters, we call them nuclei. 


\section{The Deprojected Brightness Profiles of Cuspy Cores}

Many astrophysical questions about cores require us to know the slopes of the deprojected brightness profiles. At $r \ll 0.1 r_{b}$ and $\gamma>0$, this is $-(\gamma+1)$ for the fitting function in Equation 1, but at $0.1 r_{b} \lesssim r<r_{b}$, it is considerably shallower than $-(\gamma+1)$. Our observations do not reach $r \ll 0.1 r_{b}$, so we cannot be sure that the slope is ever as steep as $-(\gamma+1)$. In any case, as Merritt (private communication) has emphasized, small departures of the observed profiles from Equation 1 are greatly magnified in deprojection, so we can be misled if we merely deproject the fit of Eq. 1 to the data. More reliable is a nonparametric deprojection of the profiles. Therefore we ask: What is the relationship between the logarithmic slopes of the projected and deprojected profiles at the smallest radii we can reach, $r \simeq 0.1 r_{b}$ ?

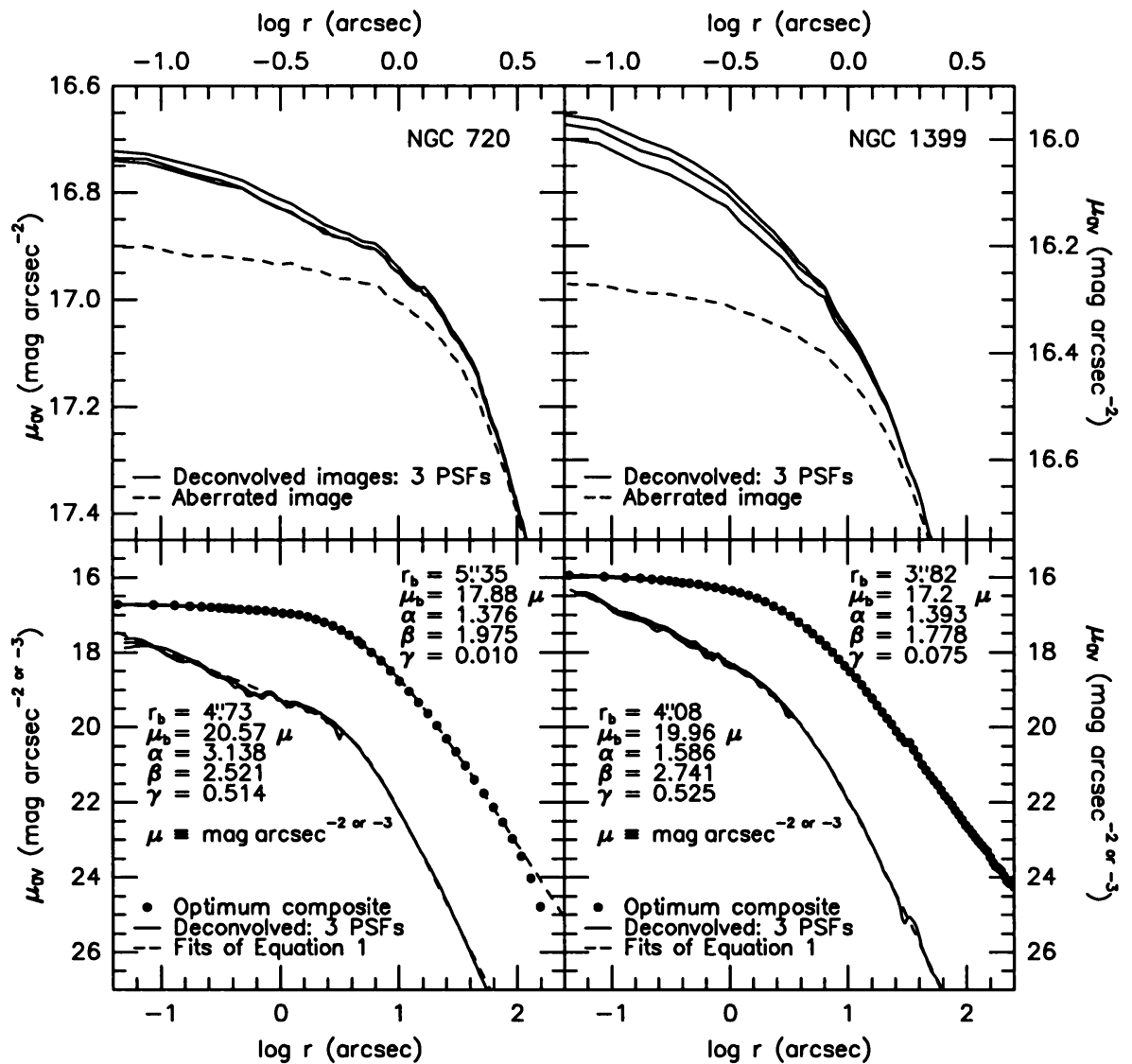

Figure 1. (top) Major-axis brightness profiles of NGC 720 and NGC 1399 before and after deconvolution with three PSFs. (bottom) The three deconvolved profiles from the top panels are shown before (above) and after (below) deprojection. Equation 1 has been fitted to the optimally deconvolved profiles (dashed lines and tabulated major-axis parameters). 


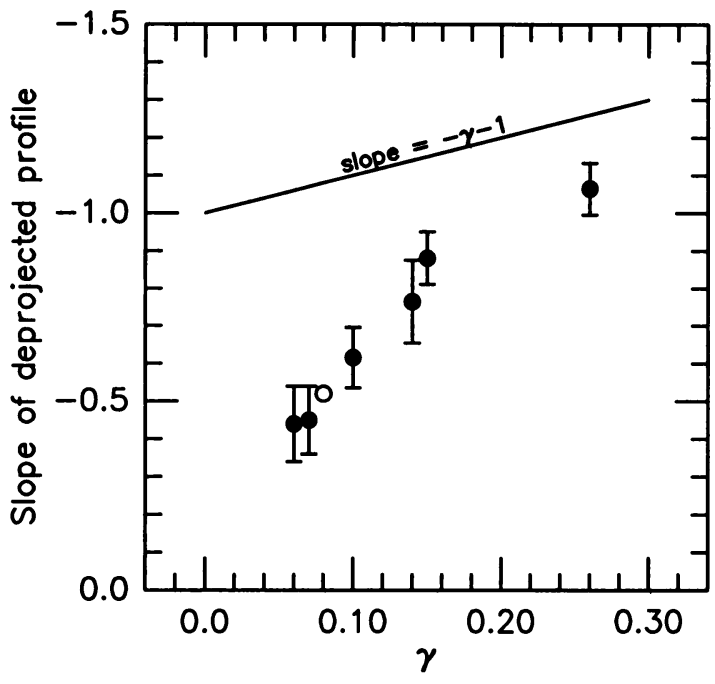

Figure 2. Correlation between the slopes of the volume and surface brightness profiles for galaxies in Lauer et al. (1995) with $r_{b}=2 . \prime 5-11 . " 1$, i.e., large enough so we can derive the profile slope at $r \simeq 0.1 r_{b}$ with confidence. From left to right, the galaxies are NGC 4889, NGC 720, NGC 6166 (open circle: the active nucleus reduces our leverage on $\gamma$ ), NGC 1399, NGC 4874, NGC 4636, and M 87. Parameters are for the major-axis profiles. Merritt \& Fridman (1995a) and Gebhardt et al. (1995) obtain similar results.

To proceed, we need to know the accuracy of the profiles. Deconvolution uncertainties dominate over photon statistics and calibration errors. So the easiest way to proceed is as follows. Over the time span of our observations ( $\sim 1.5$ years), the focus of the telescope drifted substantially. Therefore Lauer et al. (1995) used three quite different PSFs. To estimate profile errors here, images of galaxies with cuspy cores were separately deconvolved with all three PSFs. Profiles derived from these images are shown in Fig. 1. The profile obtained with the correct PSF is the bottom one. The others are derived with PSFs that are certainly wrong. So the differences between the profiles in Fig. 1 overestimate the systematic errors due to deconvolution. We conclude that profile errors are $\lesssim 0.02 \mathrm{mag} \operatorname{arcsec}^{-2}$ for the flattest cores and $\lesssim 0.05 \mathrm{mag} \operatorname{arcsec}^{-2}$ for all cuspy cores with $r_{b} \gtrsim 2^{\prime \prime}$. Figure 1 in Lauer et al. (1995) shows the analogous result for a power-law profile.

Figure 1 shows that the deprojected profiles are very nearly power laws at $r \simeq 0.1 r_{b}$. As expected, they are shallower than $\rho \propto r^{-(\gamma+1)}$. Slopes were derived by fitting power laws or Eq. 1, allowing for errors of $\leqslant 0.05 \mathrm{mag}$ $\operatorname{arcsec}^{-2}$. The results are in Fig. 2. For M 87, $\gamma$ and $r_{b}$ are large; then the slope after deprojection approaches $-(\gamma+1)$. But for flatter cores, the slopes depart more and more from $-(\gamma+1)$. The flattest profiles are quite shallow. One conclusion is that box orbits can survive: Merritt \& Fridman (1995a, b) and de Zeeuw (1995) show that cuspy cores preclude box orbits unless the deprojected profile is sufficiently shallow (approximately $\rho \propto r^{-0.5}$ ). 


\section{Fundamental Plane Parameter Correlations}

Figure 3 shows two projections of the FP correlations (Faber et al. 1987; Djorgovski, de Carvalho, \& Han 1988). Resolved cores (filled circles) satisfy FP correlations like those seen from the ground. Lower-luminosity galaxies have smaller cores of higher surface brightness. Many unresolved galaxies are consistent with the extrapolation of these correlations, but we have only upper limits on any break radii, $r_{b} \lesssim 00^{\prime \prime} 1$. These objects may have much smaller cores or they may not have cores at all.

The faintest Virgo Es look much less compact than M32. This is a resolution effect. Figure 3 shows that if M 32 were in the Virgo Cluster, the HST limits on its core parameters would be similar to those observed for the smallest ellipticals in the cluster. M 32 appears normal for its low $L$.

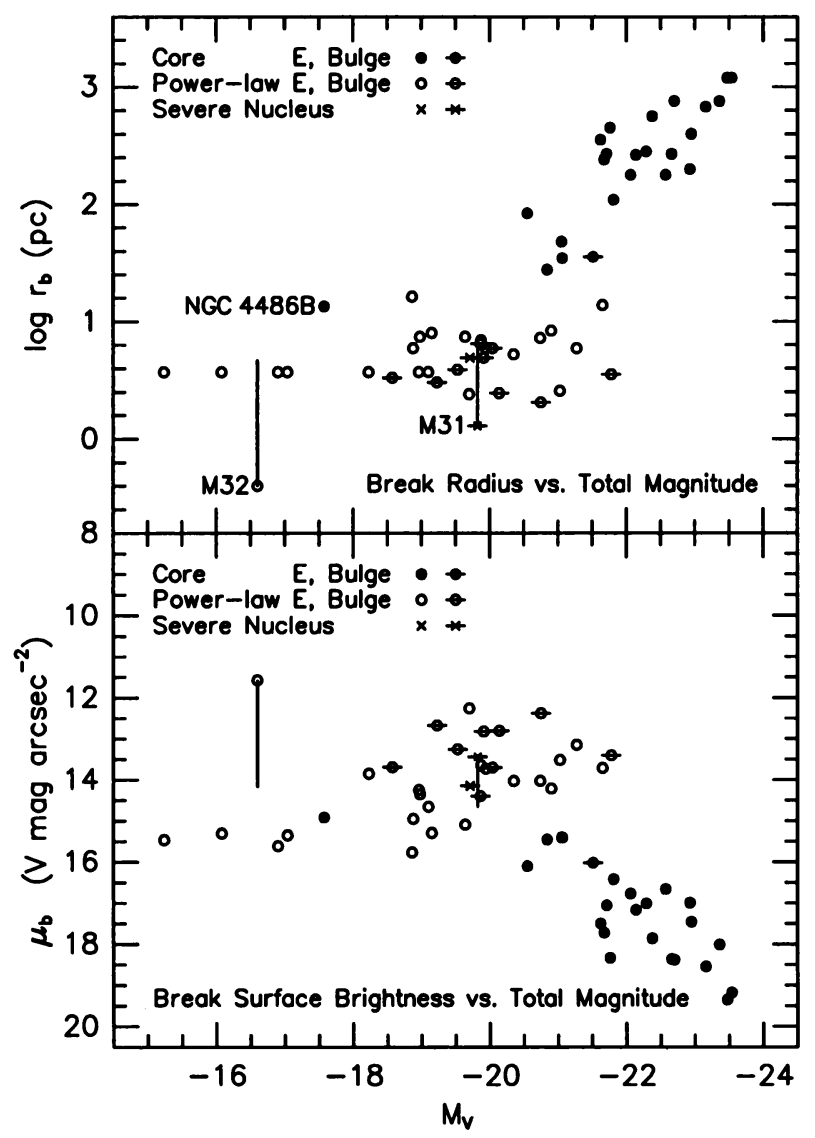

Figure 3. Correlations of $r_{b}$ and $\mu_{b}$ with absolute magnitude (from Faber et al. 1995). M 31 and M 32 are plotted twice; the symbols represent the galaxies as observed; the lines point to the parameters that we would observe if the galaxies were in the Virgo Cluster. Distances are based on a Hubble constant of $H_{0}=80 \mathrm{~km} \mathrm{~s}^{-1} \mathrm{Mpc}^{-1}$. 


\section{A Dichotomy Between Two Kinds of Elliptical Galaxies?}

At $M_{V} \simeq-21$ in Fig. 3, some galaxies have rather flat cores and others have power-law profiles. This illustrates the biggest surprise in our data. From ground-based FP correlations, we expected that marginally resolved cores would be well resolved with HST. At $M_{V} \simeq-21$, this did not happen. Most galaxies with marginal cores turned out to have power-law profiles.

As a result, the scatter in Fig. 3 is not random. Figure 4 shows the correlations of $\gamma$ with $M_{V}$ and $r_{b}$. There are signs of a dichotomy. Bright ellipticals have $0 \leq \gamma \lesssim 0.25$ (they are very well resolved); low-luminosity galaxies have $0.5 \lesssim \gamma \lesssim 1.3$ (they are very unresolved); between these, there is a gap (few galaxies are marginally resolved). The gap is especially clearcut for galaxies with large $r_{b}$ (in arcsec) (right panel). When $\gamma \gtrsim 0.5$, these have power-law profiles. A fit of Equation 1 then seizes on any small curvature in the profile and spits out a value of $r_{b}$ that has no physical meaning. Equation 1 is not well suited to deriving parameters for power-law profiles.

The possible dichotomy between two kinds of ellipticals was discovered by Nieto et al. (1991). In their sample, no elliptical with disky isophote distortions showed a core. All resolved cores were in boxy galaxies. We confirm and extend this conclusion. Symbol types in Fig. 4 encode isophote distortion (left) and the dynamical importance of rotation (right). In particular, filled squares identify galaxies that have boxy or neutral isophote distortions $\left(100 a_{4} / a \leq 0.4\right.$; see Bender 1987; Bender et al. 1989) and that rotate slowly $\left(V / \sigma^{*}<0.5\right.$; see Davies et al. 1983). Almost all power-law galaxies are disky-distorted and rotate rapidly, and almost all cores are in boxy/neutral ellipticals that rotate slowly. Slow rotation implies velocity anisotropy and triaxial structure (Illingworth 1977; Binney 1976, 1978a, b).

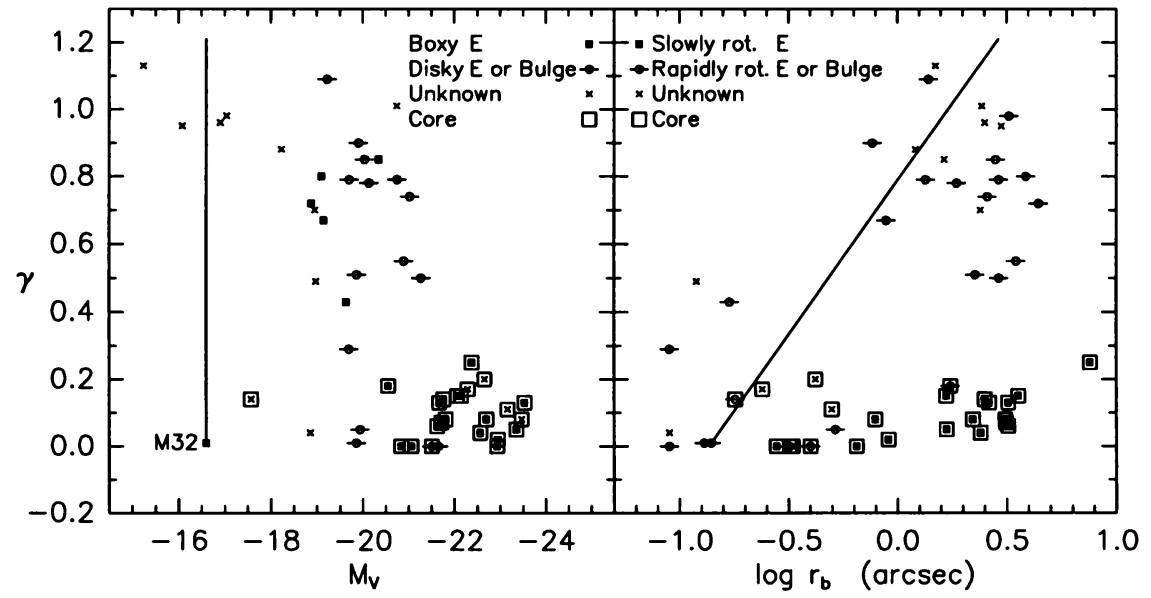

Figure 4. Inner profile slope vs. $M_{V}$ (Kormendy et al. 1994) and $r_{b}$ (Faber et al. 1995). 
Isophote distortions also prove to be diagnostic of velocity anisotropy (Kormendy \& Bender 1995). Figure 5 shows correlations of two kinematic diagnostics with $100 a_{4} / a$. Here $V / \sigma$ is the ratio of the maximum rotation velocity to the mean velocity dispersion near the center, and $(V / \sigma)^{*}$ is the ratio of $V / \sigma$ to the value expected for isotropic oblate spheroids that are flattened by rotation. I. e., $(V / \sigma)^{*} \simeq 1$ implies a nearly isotropic velocity dispersion tensor, while $(V / \sigma)^{*} \lesssim 0.5$ implies substantial anisotropy. Similarly, minor-axis rotation implies triaxiality and hence anisotropy. So: Fig. 5 shows that disky-distorted galaxies are nearly isotropic, while essentially all of the anisotropic galaxies are boxy-distorted or neutral.

Given this result, global properties of ellipticals independently suggest the same dichotomy as do the core properties (Kormendy \& Bender 1995; Kormendy \& Djorgovski 1989). If we plot galaxy ellipticity versus $100 a_{4} / a$,

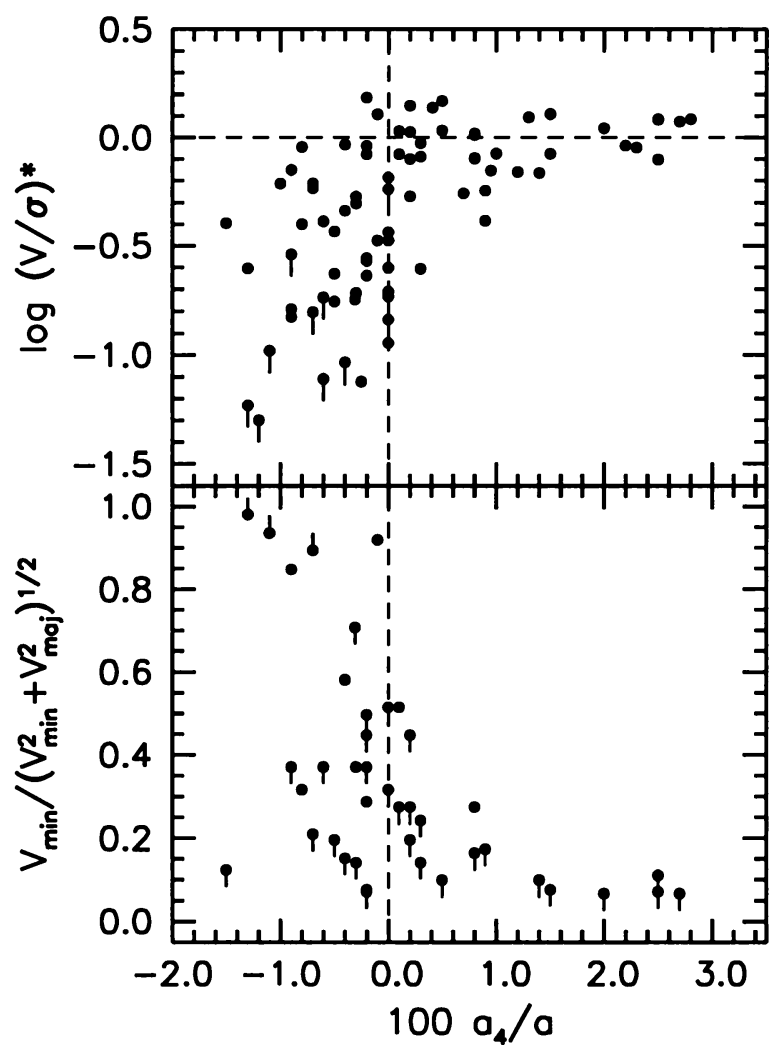

Figure 5. Correlations with isophote shape of parameters that are diagnostic of velocity anisotropy (Kormendy \& Bender 1995). Here $100 a(4) / a$ is the percent inward or outward perturbation of isophote radii along the major axis; negative values indicate boxy isophotes; positive values indicate disky isophotes. The upper panel (first illustrated in Bender 1988) shows the rotation parameter $(V / \sigma)^{*}$. The lower panel shows minor-axis rotation velocity normalized by an indicative total rotation velocity. 
we find a $\mathrm{V}$-shaped distribution. E0 galaxies have $100 a_{4} / a \simeq 0$. E4 galaxies have $100 a_{4} / a \simeq-0.8$ or $\gtrsim+1$ but not $\sim 0$. If ellipticals are mostly oblate, then spherical galaxies are rare and the most common intrinsic shape is E4 (Sandage, Freeman, \& Stokes 1970; Binney \& de Vaucouleurs 1981). This suggests that the almost-round, almost-elliptical Es are almost face-on. Edge-on Es are either substantially disky or substantially boxy. Ellipticals divide themselves into boxy, anisotropic and disky, isotropic subgroups. This led Kormendy \& Bender to propose that the Hubble sequence be revised as follows: boxy $\mathrm{E}$ - disky $\mathrm{E}-\mathrm{S} 0-\mathrm{Sa}-\mathrm{Sb}-\mathrm{Sc}$ galaxies. Then Es continue the sequence (right to left) of decreasing importance of rotation and increasing importance of random motions and velocity anisotropy.

We conclude: Core and global properties both suggest that there are two different kinds of elliptical galaxies, (i) average- and low-luminosity Es that rotate rapidly and that are nearly isotropic, approximately oblate-spheroidal, disky-distorted, and coreless, and (ii) giant ellipticals that essentially do not rotate, that are anisotropic, moderately triaxial, and boxy-distorted, and that have cuspy cores (Faber et al. 1995).

The dichotomy is suggestive but not certain. It is a subtler distinction than the one between $\mathrm{E}$ and Sph galaxies. Nevertheless, a dichotomy would suggest that two different formation processes made elliptical galaxies.

\section{Why Do Cuspy Cores Exist?}

As we explored core properties, we came to realize that neither the existence nor the survival of cores is easy to understand. Galaxy centers are vulnerable to dissipation; this builds up the central density and makes steep profiles (Mihos \& Hernquist 1994). Also, accretion of small, dense Es tends to destroy the core FP (Kormendy 1984, 1987a). Possible ways to understand cores are discussed in Faber et al. (1995). None is yet convincing. We therefore conclude with two puzzles. How did cores form? And how have they survived continued hierarchical clustering and merging?

\section{Acknowledgements}

We thank D. Merritt for urging us to determine the slopes of the deprojected profiles. This work was supported by HST data analysis funds through grant GO-02600.01-87A and by NSERC. Kormendy's ground-based work was supported by NSF grant AST 9219221.

\section{References}

Bender, R.: 1987, Mitt. Astr. Gesellschaft, No. 70, 226

Bender, R.: 1988, Astr. Ap. 193, L7

Bender, R., Burstein, D., \& Faber, S. M.: 1993, Ap. J. 411, 153 
Bender, R., Burstein, D., \& Faber, S. M.: 1994, in Panchromatic View of Galaxies Their Evolutionary Puzzle, ed. G. Hensler et al., Edit. Frontières: Gif-sur-Yvette, 99

Bender, R., et al.: 1989, Astr. Ap. 217, 35

Binggeli, B.: 1987, in Nearly Normal Galaxies: From the Planck Time to the Present, ed. S. M. Faber, Springer-Verlag: New York, 195

Binggeli, B.: 1994a, in ESO/OHP Workshop on Dwarf Galaxies, ed. G. Meylan \& P. Prugniel, ESO: Garching, 13

Binggeli, B.: 1994b, in Panchromatic View of Galaxies - Their Evolutionary Puzzle, ed. G. Hensler et al., Editions Frontières: Gif-sur-Yvette, 173

Binggeli, B., \& Cameron, L. M.: 1991, Astr. Ap. 252, 27

Binggeli, B., Sandage, A., \& Tammann, G. A.: 1988, Ann. Rev. Astr. Ap. 26, 509

Binney, J.: 1976, M. N. R. A. S. 177, 19

Binney, J.: 1978a, M. N.R. A. S. 183, 501

Binney, J.: 1978b, Comments Ap. 8, 27

Binney, J., \& de Vaucouleurs, G.: 1981, M. N. R. A. S. 194, 679

Byun, Y.-I., et al.: 1995, A. J., in preparation

Crane, P., et al.: 1993, A. J. 106, 1371

Da Costa, G. S.: 1992, in IAU Symposium 149, The Stellar Populations of Galaxies, ed. B. Barbuy \& A. Renzini, Kluwer: Dordrecht, 191

Davies, R. L., et al.: 1983, Ap. J. 266, 41

de Zeeuw, T.: 1995, in IAU Symposium 171, New Light on Galaxy Evolution, ed. R. L. Davies \& R. Bender, Kluwer: Dordrecht, in press

Djorgovski, S., de Carvalho, R., \& Han, M.-S. 1988: in The Extragalactic Distance Scale, ed. S. van den Bergh \& C. J. Pritchet, ASP: San Francisco, 329

Djorgovski, S. G., Pahre, M. A., \& de Carvalho, R. R.: 1995, in Fresh Views of Elliptical Galaxies, ed. A. Buzzoni et al., ASP: San Francisco, in press

Djorgovski, S., \& Santiago, B. X.: 1993, ESO/EIPC Workshop: Structure, Dynamics, and Chemical Evolution of Early-Type Galaxies, ed. J. Danziger et al., ESO: Garching, 59

Faber, S. M., et al.: 1987, in Nearly Normal Galaxies: From the Planck Time to the Present, ed. S. M. Faber, Springer-Verlag: New York, 175

Faber, S. M., et al.: 1995, A. J., in preparation

Ferguson, H. C., \& Binggeli, B.: 1994, Astr. Ap. Rev. 6, 67

Ferguson, H. C., \& Sandage, A.: 1991, A. J. 101, 765

Ferrarese, L., et al.: 1994, A. J. 108, 1598

Forbes, D. A.: 1994, A. J. 107, 2017

Forbes, D. A., Franx, M., \& Illingworth, G. D.: 1994, Ap. J. 428, L49

Forbes, D. A., Franx, M., \& Illingworth, G. D.: 1995, A. J. 109, 1988

Gebhardt, K., et al.: 1995, A. J., in preparation

Grillmair, C. J., et al.: 1994, A. J. 108, 102

Ichikawa, S.-I., et al.: 1988, A. J. 96, 62

Ichikawa, S.-I., Wakamatsu, K.-I., \& Okamura, S.: 1986, Ap. J. Suppl. 60, 475

Illingworth, G.: 1977, Ap. J. 218, L43

Jaffe, W., et al.: 1994, A. J. 108, 1567

Kormendy, J.: 1982, in Morphology and Dynamics of Galaxies, Twelfth Saas-Fee Course, eds. L. Martinet \& M. Mayor, Geneva Observatory: Sauverny, 113

Kormendy, J.: 1984, Ap. J. 287, 577

Kormendy, J.: 1985a, Ap. J. 292, L9

Kormendy, J.: 1985b, Ap. J. 295, 73

Kormendy, J.: 1987a, in IAU Symposium 127, Structure and Dynamics of Elliptical Galaxies, ed. T. de Zeeuw, Reidel: Dordrecht, 17

Kormendy, J.: 1987b, in Nearly Normal Galaxies: From the Planck Time to the Present, ed. S. M. Faber, Springer-Verlag: New York, 163

Kormendy, J., \& Bender, R.: 1994, in ESO/OHP Workshop on Dwarf Galaxies, ed. G. Meylan \& P. Prugniel, ESO: Garching, 161

Kormendy, J., \& Bender, R.: 1995. A. J., submitted 
Kormendy, J., \& Djorgovski, S.: 1989, Ann. Rev. Astr. Ap. 27, 235

Kormendy, J., et al.: 1994, in ESO/OHP Workshop on Dwarf Galaxies, ed. G. Meylan \& P. Prugniel, ESO: Garching, 147

Kormendy, J., \& McClure, R. D.: 1993, A. J. 105, 1793

Lauer, T. R.: 1985a, Ap. J. Suppl. 57, 473

Lauer, T. R.: 1985b, Ap. J. 292, 104

Lauer, T. R., et al.: 1991, Ap. J. 369, L41

Lauer, T. R., et al.: 1992a, A. J. 103, 703

Lauer, T. R., et al.: 1992b, A. J. 104, 552

Lauer, T. R., et al.: 1993, A. J. 106, 1436

Lauer, T. R., et al.: 1995, A. J. in press

Merritt, D., \& Fridman, T.: 1995a, in Fresh Views of Elliptical Galaxies, ed. A. Buzzoni et al., ASP: San Francisco, in press

Merritt, D., \& Fridman, T.: 1995b, Ap. J, submitted

Mihos, J. C., \& Hernquist, L.: 1994, Ap. J. 437, L47

Møller, P., Stiavelli, M., \& Zeilinger, W. W.: 1995, M. N. R. A. S, in press

Nieto, J.-L., Bender, R., \& Surma, P.: 1991, Astr. Ap. 244, L37

Saglia, R. P., Bender, R., \& Dressler, A.: 1993, Astr. Ap. 279, 75

Sandage, A., Binggeli, B., \& Tammann, G. A.: 1985, A. J. 90, 1759

Sandage, A., Freeman, K. C., \& Stokes, N. R.: 1970, Ap. J. 160, 831

Schweizer, F.: 1980, Ap. J. 237, 303

Schweizer, F.: 1981, Ap. J. 246, 722

Stiavelli, M., Møller, P., \& Zeilinger, W. W.: 1993, Astr. Ap. 277, 421

Tremaine, S.: 1995, in Some Unsolved Problems in Astrophysics, in press

van den Bosch, F. C., et al.: 1994, A. J. 108, 1579

Young, P. J., et al.: 1978, Ap. J. 221, 721

Wirth, A., \& Gallagher, J. S.: 1984, Ap. J. 282, 85

\section{Discussion}

A. Renzini: As you pointed out, faint galaxies have such high phase space densities that they should easily survive if accreted by bright ellipticals. The fact that bright ellipticals don't have power-law nuclei seems to argue against recent merging, doesn't it?

J. Kormendy: Yes, that is precisely our point. There are rare exceptions, like NGC 1316, in which a steep density profile is seen and in which there is evidence for a recent merger (Schweizer 1980, 1981; Kormendy 1987a). But in general, mergers of present-day ellipticals tend to destroy the core FP relations unless some process can heat the core. One possibility is binary black holes, perhaps themselves a result of the merger (Faber et al. 1995). W. Dehnen: A way to solve the problem of forming giant Es from lowluminosity Es may be to make the cuspy cores later by secular evolution. Do you see any signs of secular evolution (e.g., barlike distortions)?

J. Kormendy: The secular process for which we see evidence is accretion of gas-rich fragments. We see central dust disks, stellar disks (sometimes made of young stars), and in one case, both a dust disk and a stellar disk (Kormendy et al. 1994). Accretion tends to increase the central density and fill in any cores. The only galaxy in which we see an elongated center (actually an asymmetric one, as in M 31) is NGC 4486B. 